\title{
ERS International Congress 2020 Virtual: highlights from the Respiratory Intensive Care Assembly
}

\author{
Camille Rolland-Debord ${ }^{1}$, Alexander D'Haenens ${ }^{2}$, Leire Mendiluce ${ }^{3}$, Lydia Spurr ${ }^{4}$, Shruthi Konda ${ }^{5}$, \\ Radostina Cherneva ${ }^{6}$, Elodie Lhuillier ${ }^{7}$, Leo Heunks ${ }^{8}$ and Maxime Patout $\mathbb{C}^{9,10}$
}

${ }^{1}$ AP-HP, Groupe Hospitalier Universitaire APHP-Sorbonne Université, Service des Explorations Fonctionnelles de la Respiration de l'Exercice et de la Dyspnée, Hôpital Tenon, Paris, France. ${ }^{2}$ Dept of Respiratory Medicine, University Hospitals Leuven, Leuven, Belgium. ${ }^{3}$ Ventilation Unit and Respiratory Semi-Critical Care Unit, Dept of Respiratory Medicine, University Hospital Germans Trias i Pujol, Universitat de Barcelona, Barcelona, Spain. ${ }^{4}$ Academic and Clinical Dept of Sleep and Breathing, Royal Brompton and Harefield Hospitals, London, UK. ${ }^{5}$ Dept of Respiratory Medicine, Royal Brompton Hospital, London, UK. ${ }^{6}$ Medical University, Sofia, Dept of Respiratory Diseases, University Hospital 'St Sophia', Sofia, Bulgaria. ${ }^{7}$ Unité de recherche clinique, Centre Henri Becquerel, Rouen, France. ${ }^{8}$ Dept of Intensive Care, Amsterdam University Medical Center, Amsterdam, the Netherlands. ${ }^{9}$ AP-HP, Groupe Hospitalier Universitaire APHP-Sorbonne Université, Service des Pathologies du Sommeil (Département R3S), Paris, France. ${ }^{10}$ Sorbonne Université, INSERM, UMRS1158 Neurophysiologie Respiratoire Expérimentale et Clinique, Paris, France.

Corresponding author: Maxime Patout (maxime.patout@aphp.fr)

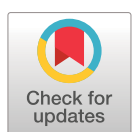

This version is distributed under the terms of the Creative Commons Attribution NonCommercial Licence 4.0. For commercial reproduction rights and permissions contact permissions@ersnet.org

Received: 25 March 2021 Accepted: 19 Aug 2021

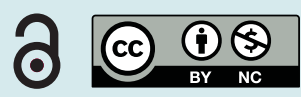

Shareable abstract (@ERSpublications)

Updates from \#ERSCongress 2020 on diagnostic strategies in patients admitted to the ICU with acute respiratory failure and on the management of \#SARSCoV2 infection https://bit.ly/38cx0Pi

Cite this article as: Rolland-Debord C, D'Haenens A, Mendiluce L, et al. ERS International Congress 2020 Virtual: highlights from the Respiratory Intensive Care Assembly. ERJ Open Res 2021; 7: 002142021 [DOI: 10.1183/23120541.00214-2021].

\section{Abstract}

During the virtual European Respiratory Society Congress 2020, early career members summarised the sessions organised by the Respiratory Intensive Care Assembly. The topics covered included diagnostic strategies in patients admitted to the intensive care unit with acute respiratory failure, with a focus on patients with interstitial lung disease and for obvious reasons, severe acute respiratory syndrome coronavirus 2 (SARS-CoV-2) infection. These sessions are summarised in this article, with take-home messages highlighted.

\section{State-of-the-art session: respiratory critical care}

The role of chest computed tomography scanning in the differential diagnosis of acute respiratory failure in ventilated patients (M. Prokop, Nijmegen, the Netherlands)

According to M. Prokop, chest computed tomography (CT) scans can be used as a "problem solver" to identify causes of acute respiratory failure (ARF) when a diagnosis cannot be established from the initial chest radiograph or ultrasound. M. Prokop was supportive of the use of inspiratory chest CT scans with a narrow slice width of 0.5 to $1 \mathrm{~mm}$, multiplane reconstructions and intravenous contrast injection. Intravenous contrast differentiates pleural disease from intrapulmonary disease, e.g. atelectasis from consolidation. He also suggested the use of expiratory chest CT scans in cases where air trapping is suspected. The speaker reminded us that, except in low-dose CT, all contemporary chest CT scans now create high-resolution images and use radiation doses lower than natural background radiation (except for larger patients).

Different clinical situations were then described by M Prokop: on a chest radiograph, the absence of air bronchogram does not indicate extrapulmonary disease, and the volume of a pleural effusion must be greater than $200 \mathrm{~mL}$ to be visualised. In pleural effusions, ultrasound predicts drainage success better than CT $[1,2]$. However, chest scans can differentiate empyema (obtuse angle with the pleura) from subpleural abscesses (acute angle); bronchopleural fistula is a complication of pneumonia and may be suspected if air is observed within areas of in the consolidation. Chest CT scans are also the diagnostic gold standard for pulmonary embolism, and can be useful in estimating the size and extent of pneumothoraces or 
barotrauma, including in acute respiratory distress syndrome (ARDS), and in visualising interstitial and mediastinal emphysema.

However, chest CT scans are of modest utility in discriminating between other clinical conditions, e.g. between oedema, pneumonia, ARDS and organising pneumonia, and in such cases additional clinical information is required. However, ventral consolidations are more typical for pneumonia, and symmetrical or moving consolidations are more suggestive of oedema.

\section{Take-home messages}

- Chest CT scans are excellent in distinguishing atelectasis from other causes of consolidation, in distinguishing consolidations and pleural from parenchymal disease, and in the diagnosis of pulmonary embolism and infarct.

- Chest CT scans are of modest utility in identifying the cause of consolidation (e.g. organising pneumonia, pulmonary haemorrhage, ARDS, oedema).

- Chest CT scans are less useful in the differential diagnosis of pneumonia and predicting treatment outcomes of complex pleural effusions.

Role of bronchoalveolar lavage and lung biopsy in the diagnosis of ARF in intensive care unit patients (V. Poletti, Forli, Italy)

V. Poletti discussed that the most common causes of ARF are ARDS and infections, but the two entities can overlap as infections can cause ARDS. There are histopathological differences between ARDS and other entities that may mimic it, such as idiopathic acute interstitial pneumonia, acute exacerbation of interstitial lung disease (ILD), organising pneumonia, anti-glomerular basement membrane antibody disease, granulomatosis with polyangiitis, diffuse alveolar haemorrhage, acute hypersensitivity pneumonitis and acute hypereosinophilic pneumonia [3].

In patients with hypoxic respiratory failure, a bronchoscopy with bronchoalveolar lavage (BAL) can be considered only after clinical, biological and radiological assessments have been performed. The most frequent side-effects are fever and hypoxia, especially with large volume instillations. Rarely complications occur, such as hypoxaemia, hypotension, bradycardia, haemoptysis, pneumothorax, bronchospasm, bleeding, Takotsubo cardiomyopathy and acute exacerbations of ILD [4].

Diffuse alveolar damage (DAD) is the histological term used to describe specific changes that occur during acute lung injury. The pathological alterations observed in DAD vary depending on the interval between the onset of symptoms and the time of cyto-histological lung sampling. The early exudative stage of DAD is characterised by oedema and hyaline membrane formation. This progresses to a proliferative stage in which there is hyperplasia of type II pneumocytes, desquamation of alveolar lining cells, and thickening of the interstitium with fibroblast proliferation. Fibrosis may then occur with architectural destruction and honeycombing.

The cytopathological profile for DAD consists of more than 85\% neutrophils, atypical type II pneumocytes and amorphous extracellular material (hyaline membranes). Cytological profiles that differ from this neutrophilic-predominant pattern should suggest ARDS mimics, for example diffuse alveolar haemorrhage (blood-stained BAL fluid, haemosiderin loaded macrophages) or disseminated malignancy [5]. Microbiological testing may identify a treatable cause of ARDS and has a high diagnostic yield in the immunocompromised patient [6].

Lung biopsy should be considered when BAL does not lead to a diagnosis. The decision to perform a lung biopsy should be made based on lung compliance and oxygenation, and should consider whether specific procedures and therapies are available, and the anticipated time to biopsy. DAD is present in the majority of patients with ARF secondary to nonresolving ARDS and the frequency of DAD does not differ across the three stages of ARDS [7]. Although some studies show that open lung biopsy (OLB) provides a specific diagnosis in up to $84 \%$ of patients and leads to a change in management in up to $73 \%$ of cases, the risk of complications is unclear with only two studies reporting complication rates: these varied from $0 \%$ to $56 \%[8,9]$. The wide range may be explained by differences in patient characteristics and definitions of complications. ARF studies did not compare differences in mortality between those who had an OLB and those who did not. Alternatives to OLB are transbronchial (cryo)biopsy. Limited data suggest that they have a high diagnostic yield with fewer complications than OLB [10, 11]. 
Take-home messages

- BAL can identify a specific diagnosis in a minority of cases, but the greatest yield is in alveolar filling processes. It is a relatively low-risk technique in acutely ill patients.

- When no diagnosis is made after BAL, lung biopsy may be indicated, but it is mandatory to discuss this on a case-by-case basis, taking into account the risk-benefit ratio.

- Limited data suggest fewer complications in transbronchial cryobiopsy compared with OLB.

The future of pulmonary diagnostics in intensive care unit patients (L. Bos Amsterdam, the Netherlands)

L. Bos first presented the diagnostic challenges in intensive care, i.e., identifying the cause of respiratory failure, understanding why a patient's lung injury is not resolving, and knowing if the patient is developing pneumonia.

Lung ultrasound (LUS), currently used as a dichotomous diagnostic test, should be used more quantitatively, for example to describe the size of pleural effusions or the amount of pulmonary oedema. Quantification of B-lines, either by manual scoring or by a computerised preprocessing algorithm, correlates very well with measurements of extravascular lung water [12]. The effect of an intervention can also be monitored by dynamic LUS. Scoring for lung reaeration by chest CT strongly correlates with scoring by LUS, suggesting that the latter could be used as a bedside tool to monitor responses to changes of ventilatory settings [13]. Another promising technique is the use of contrast-enhanced LUS to examine peripheral consolidations: hypoechoic areas suggest the absence of perfusion and the possibility of infarction [14].

Confocal lung endomicroscopy (CLE) is an imaging technique using a laser-based bronchoscopic intervention that assesses the alveolar morphology in situ by detection of elastin in the extracellular matrix. As it is difficult to differentiate between oedema, inflammation and underlying fibrosis on CT, CLE can help by showing distortion of normal architecture, which is particularly prominent in fibrosis [15].

Another promising tool is biological assessment. Breath contains hundreds of volatile metabolites that can be reflective of pulmonary infection, pulmonary inflammation, and/or oxidative stress response. A rapid bedside breath analysis by gas chromatography and mass spectrometry shows potential as a biomarker in the diagnosis of ARDS and the exclusion of bacterial causes for nosocomial pneumonia [16, 17].

Several "omics" techniques have been used to better phenotype patients and to provide rapid results. The broad use of molecular techniques could improve diagnostic yield. Real-time metagenomic sequencing of BAL fluid seems to be a more sensitive and faster way to identify the causative pathogen in pneumonia compared with standard cultures $[18,19]$. It can also show the diversity of the lung microbiome, which predicts intensive care outcomes [20]. The use of transcriptomic profiles has shown that inflammatory markers in the blood are not very well correlated with markers in the lung. This is an additional argument to focus our diagnostic sampling on the lung [21]. These techniques can also give us more data about the host response to inflammation.

\section{Take-home messages}

- LUS has the advantage of instant clinical feedback and is easily repeatable.

- Confocal lung endoscopy detects in vivo pathology and is easily repeatable. However, it only provides a focal assessment and requires bronchoscopy.

- Breath analysis provides rapid results, but lacks specificity with sampling errors

- Comprehensive "omics" techniques are likely to be more accurate in providing a comprehensive snapshot of disease but are time/labour intensive and require sampling of the lower airway.

Hot topics: ILD patients in the intensive care unit

Diagnostic procedures in intensive care unit: bronchoscopy and BAL or tissue? (C. Ravaglia, Forli, Italy)

Hypoxic respiratory failure is a frequent cause of admission to the intensive care unit (ICU) and contributes significantly to morbidity and mortality. Diagnosis of lung infiltrates of unclear aetiology is a major challenge in ICU [22, 23]. BAL may be performed when it is not possible to retain a definite diagnosis or identify a specific cause after taking a complete history, physical examination, global clinical assessment, chest CT scan, laboratory tests (including immunological and serological testing), echocardiography and/or pulmonary artery catheterisation. BAL is a relatively low risk technique, which is 
well tolerated, easily performed with flexible bronchoscopy, and usually safely performed in acutely ill patients [24].

Rapid on-site cytological examination of BAL fluid may have a role in determining specific diagnoses [25]. In patients with long-standing ILDs with new onset lung infiltrates admitted to ICU with ARF, BAL may be diagnostic only in a minority of cases (13\%), and hospital mortality and therapeutic management appear to be unchanged regardless of BAL findings [26]. The greatest yield for BAL is obtained in patients with pathological alveolar filling processes (such as some opportunistic infections, peripheral malignancies, alveolar proteinosis, alveolar haemorrhage, lipoid pneumonia, etc.).

Cytological analysis on BAL fluid is most useful for lung malignancies (particularly for adenocarcinoma or tumours with lymphangitic growth patterns) and haematologic malignancies. Morphological analysis may be implemented by immunocytochemical or molecular tests to identify the cell lineage and the presence of monoclonality [5]. Total white cell count, differential cell counts, lymphocyte subpopulations and immunohistochemistry are mostly used to identify ILDs and lymphomatous or leukaemic infiltrates.

As there are no established guidelines, the decision to perform a lung biopsy should be discussed during multidisciplinary meetings and answer the following questions. 1) Could the biopsy provide a specific diagnosis which is of benefit to the patient? 2) Could the patient tolerate further insult to lung compliance and oxygenation? The timing and procedure used to perform the biopsy should also be discussed [27].

OLB is rarely performed in ICU patients because of the poor physiological conditions of most patients and because DAD has traditionally been considered the pathological signature of ARDS, thus making the biopsy appear less necessary [28]. However, in reality the sensitivity and specificity of DAD for ARDS is low [7]. The most frequent therapeutic change implemented after biopsy is the decision to initiate, continue, adjust the dose, or stop the administration of steroids, but management of antimicrobials and end-of-life decision making are also important $[8,9]$.

There is a need for a prospective, multicentre, randomised controlled trial evaluating the survival benefit of lung biopsy for patients with ILD, ARF and lung infiltrates.

Take-home messages

- Bronchoscopy is a relatively low-risk technique. It may have an important role in the ICU, particularly in the diagnosis of alveolar filling processes.

- When no definite diagnosis is obtained after BAL, lung biopsy may be indicated; however, there are no widely accepted guidelines and there is an urgent need for a prospective randomised controlled trial evaluating the real-world benefit of lung biopsy for these patients.

- Surgical lung biopsy may provide a specific diagnosis and lead to a change in management in the majority of patients.

Radiological patterns in ICU: does it matter? (N. Sverzellati, Parma, Italy)

Patients admitted with ARF should be assessed as to whether the condition could be related to undiagnosed ILD. Assessment should consider clinical risk factors for progression of interstitial lung abnormalities (ILA) (e.g. cigarette smoking), inhalational exposure, medications (e.g. chemotherapy, immune checkpoint inhibitors), radiation therapy, and thoracic surgery. When analysing the chest CT, physicians should assess patterns associated with progressive disease, i.e., the presence of non-fibrotic ILA with basal and peripheral predominance, and fibrotic ILA with basal and peripheral predominance with or without honeycombing.

Previously undiagnosed usual interstitial pneumonia (UIP) is identified in 50\% of patients dying from acute interstitial pneumonia [29]. 59\% of patients with ARF complicating ILD do not have a pre-morbid diagnosis of ILD prior to ICU admission [30]. Signs of fibrosis on CT are associated with increased in-hospital mortality, 1-year mortality and failure to respond to high-dose corticosteroids.

In ARF, honeycombing is a better diagnostic marker than bronchiectasis for underlying UIP, because of the difficulty discriminating irreversible traction bronchiectasis from free standing bronchiectasis during acute lung injury. Analysis of prior radiological examinations (even chest radiographs) is particularly helpful, if available. 


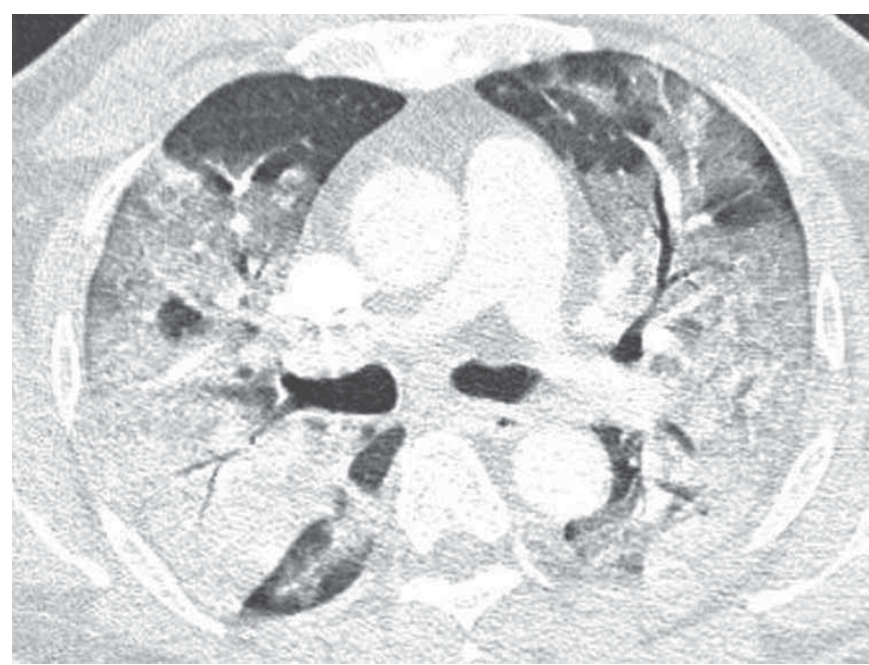

FIGURE 1 Chest computed tomography in a patient admitted to the intensive care unit for acute respiratory failure secondary to eosinophilic acute pneumonia.

Similar CT findings may be caused by different aetiologies. Interlobular septal thickening, tree-in-bud, cavitation, and features of organising pneumonia are good diagnostic markers of organising pneumonia, while ground-glass opacification is less specific (figure 1).

Extension and distribution of ground-glass opacities are associated with poorer prognosis [31]. Asymmetric distribution of acute abnormalities is associated with better prognosis; 180-day mortality was lower in patients with asymmetrical distribution compared with those in which acute abnormalities were symmetrical [32].

\section{Take-home messages}

- UIP is often not diagnosed before acute exacerbation.

- Prior chest imaging, for example, CT scans are pivotal for interpretation of acute abnormalities.

\section{Selection of ILD patients for ICU and ECMO (C. Agerstrand, New York, USA)}

The CESAR trial [33] recruited adults with severe ARF and randomised them to conventional care at their local hospital or referral and transfer to an extracorporeal membrane oxygenation (ECMO) centre. The authors met their primary outcome of an improvement in survival without severe disability at 6 months in the ECMO-referred group: 63\% versus 47\% (RR 0.69 (CI 0.05-0.97), p=0.03). However, not all patients in the ECMO group actually received ECMO, as some improved with conventional management in the tertiary care centre to which they were referred, In addition, not all patients randomised to the control group received lung-protective ventilation strategies that are known to improve outcomes in ARDS.

The EOLIA trial [34] sought to address the limitations of the pragmatically designed CESAR trial, and recruited 249 adults with severe ARDS and randomised them to a protocolised approach to mechanical ventilation or ECMO with an ultra-lung protective ventilatory approach. Using ECMO in conjunction with ultra-lung protective ventilation, 60-day mortality was not significantly lower with ECMO (35\%) than with a strategy of conventional mechanical ventilation (46\%) (relative risk 0.76 (95\% CI 0.55-1.04), p=0.09). However, some patients in the conventional mechanical ventilation group also received ECMO as rescue therapy.

A retrospective analysis included 40 patients with ILD referred to ICU for ARF treated with or without ECMO. The data suggest that ECMO is a lifesaving option for patients with ILD and ARF provided that they are candidates for lung transplantation. Indeed, ECMO was not able to improve the poor prognosis in patients that did not qualify for lung transplantation [35].

Various ECMO configurations can provide varying levels of support by making adjustments to oxygen delivery to the brain/coronary circulation and the level of haemodynamic support [36]. Venovenous 
ECMO can be used in the absence of concomitant pulmonary hypertension; venoarterial ECMO is better for patients with pulmonary hypertension.

In order to decide whether or not to start ECMO, several questions need to be answered, such as the rehabilitation potential of the patient pre- and post-ECMO, as well as factors that affect the time to potential lung transplantation (e.g. extremes of height and weight, or rare blood type that may indicate the wait will be longer). Upper body venoarterial ECMO via the subclavian or innominate artery cannulation is advised in patients on a bridge to transplant ECMO [37], as it allows ambulatory ECMO that supports physical reconditioning.

Take-home messages

- ECMO may be beneficial in ILD exacerbations as a bridge to transplantation.

- Early referral to a transplant centre is essential for potentially eligible patients.

Management of ILD patients in ICU (M. Kokosi, London, UK)

Patients with idiopathic pulmonary fibrosis (IPF) have a significantly worse prognosis at 90 days when compared with patients with non-IPF idiopathic interstitial pneumonia (IIP) or connective tissue disease (CTD)-related ILD (which have similar outcomes to each other) [38].

ILD patients admitted to the ICU can be divided in three main categories: 1) ILD with a clear autoimmune background or drug-induced ILD; 2) ILD with no autoimmune features; and 3) patients with signs of autoimmune dysregulation but no clear diagnosis. The most common ILDs seen in ICU are IIPs, CTD-related ILD, vasculitis, drug-induced ILD and acute eosinophilic pneumonia. Patients with IPF and overt fibrotic disease have poor outcomes [39, 40].

Patients with ILD admitted to ICU can also be divided into three phenotypes: phenotype A are progressive with potential for full reversibility; phenotype B are progressive with potential for partial reversibility; and phenotype $\mathrm{C}$ are rapidly progressive with unpredictable outcomes. Reversibility in an acute exacerbation of ILD is more likely with de novo ILD, features of organising pneumonia on CT, a shorter time from diagnosis, antibody positivity (apart from melanoma-differentiation-associated protein 5 (MDA5)), increased inflammatory markers and response to previous treatment. Organising pneumonia with no radiological evidence of fibrosis could be treated with i.v. methylprednisolone monotherapy (phenotype A). In contrast, a patient with Jo-1 positive inflammatory myopathy or organising pneumonia with established fibrosis would need dual therapy with i.v. methylprednisolone and cyclophosphamide or rituximab (phenotype B). Patients with MDA5 myositis (phenotype C) would need triple therapy with i.v. methylprednisolone, cyclophosphamide and rituximab. In these cases, the addition of tacrolimus, cyclosporin, or even plasmapheresis could be considered.

\section{Take-home messages}

- Prognosis of ILD in the ICU is poor, but most case series consist of mixed ILD including IPF.

- Careful selection of ILD patients for ICU admission is crucial, with involvement of ILD specialists in multidisciplinary discussions.

- The evaluation of reversibility is the cornerstone in the management of these patients

\section{Management of COVID-19}

These sessions discussed the most up-to-date advice at the time of the European Respiratory Society (ERS) Congress for the clinical management of patients with coronavirus disease 2019 (COVID-19), including respiratory support and pharmacological management. Some knowledge may have now evolved.

\section{Respiratory support (pre-ventilation, ventilation and ECMO) (P. Navalesi, Padova, Italy)}

The severe acute respiratory syndrome coronavirus 2 (SARS-CoV-2) pandemic resulted in unprecedented pressure on intensive care resources and bed capacity. In this context, the use of noninvasive respiratory support outside ICU has been demonstrated to be safe and effective [41], although it may contribute to viral aerosolisation [41], which needs to be reduced (figure 2). Successful treatment with high-flow nasal cannula (HFNC), continuous positive airway pressure (CPAP) or noninvasive ventilation (NIV) was more frequent in younger, less hypoxic patients with fewer comorbidities [41]. No significant differences in negative outcomes, including mortality and endotracheal intubation (ETI) rate, have been identified based on the noninvasive modality used [41]. However, disease severity and ceilings of care may independently affect modality selection and outcomes. 
Once a patient is placed on a ventilator, the key challenge is to avoid complications. Mechanical ventilation (in and of itself) does not produce lung healing, it merely keeps patients alive until their own biological mechanisms are able to recover. The objective is to avoid further lung inflammation [42].

Where ETI is required, individualisation of ventilatory support with the aim of preventing further complications is advised [42]. Lung-protective ventilation using conservative tidal volumes and driving pressures has been recommended [43]. Determining patients' optimal ventilatory pressures is complicated by heterogeneity in lung recruitability [44], and patient factors including comorbidities such as obesity (figure 3) [45, 46]. P. Navalesi suggested the following three stages: 1) the use of lung-protective ventilation; 2) early prone positioning, i.e., positioning within $72 \mathrm{~h}$ of ETI with a first cycle of $16 \mathrm{~h}$, then $12 \mathrm{~h}$; and 3) if there is a lack of improvement, to contact an ECMO referral centre. Further investigation is required to inform timing of weaning from invasive ventilation and extubation.

In SARS-CoV-2-induced ARDS, ventilation/perfusion ( $\left.V^{\prime} / Q^{\prime}\right)$ mismatching is typically exacerbated by hypercoagulability and development of pulmonary microthrombosis [47, 48]. Prone positioning may increase oxygenation by reducing $V^{\prime} / Q^{\prime}$ mismatching [49], but patient response is variable due to differences in disease phenotype and pathophysiology.

World Health Organization (WHO) guidelines recommend the consideration of ECMO in patients with refractory hypoxaemia despite lung-protective ventilation. Outcomes of ECMO-rescued patients appear similar in SARS-CoV-2 infection compared with other causes of ARDS [50]. It is also important to consider the practical and ethical implications for healthcare services, and the wider patient population [51].

Take-home messages

- Use of noninvasive respiratory support to manage hypoxaemia in selected patients with SARS-CoV-2 infection may prevent requirement for intubation.

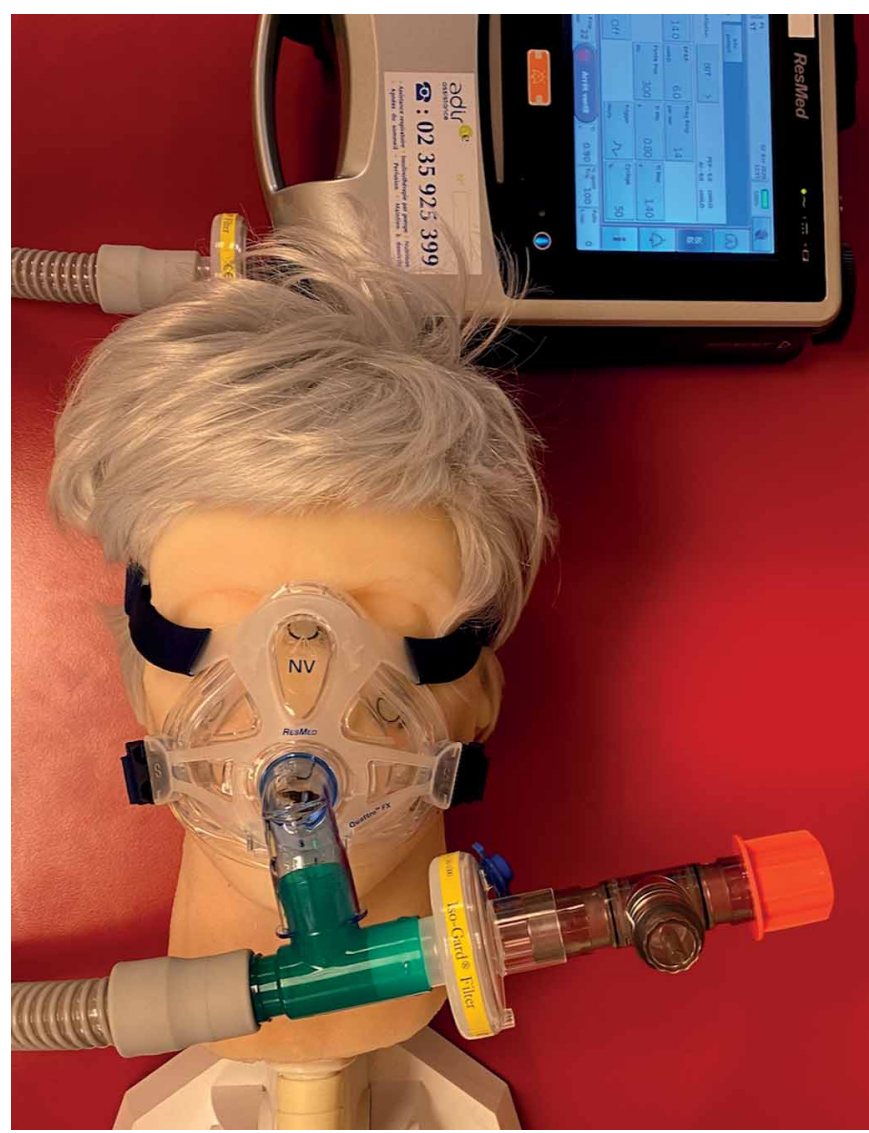

FIGURE 2 A noninvasive ventilation circuit used to minimise viral aerosolization. Reproduced from and evaluated in [79]. 


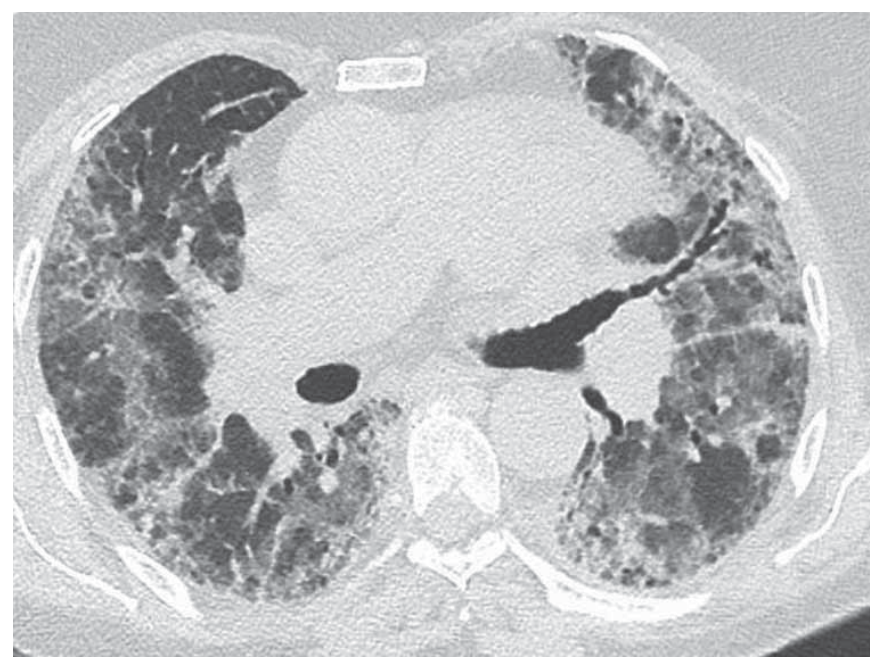

FIGURE 3 Chest computed tomography in a patient admitted to the intensive care unit for acute respiratory failure secondary to SARS-CoV-2 infection.

- Pathophysiological differences exist between patients with ARF and ARDS due to SARS-CoV-2 infection and it is essential to tailor supportive respiratory management to the individual.

Drugs: anti-virals (J. Beigel, Baltimore, USA)

J. Beigel reported the preliminary data from two trials: 1) RECOVERY (Randomised Evaluation of COVid-19 thERapY) and 2) ACTT-1 (Adaptive Covid-19 Treatment Trial).

Remdesivir

Remdesivir was one of the first antiviral treatments assessed in SARS-CoV-2 infection. Results from the ACTT-1 study were presented [52]. The study was a double-blind, randomised, placebo-controlled trial of remdesivir versus placebo for up to 10 days. Preliminary results came from 538 patients randomised to remdesivir and 521 to placebo. In patients with mild-to-moderate disease, time to recovery was 5 days for both remdesivir and placebo. In patients with severe disease, time to recovery was 12 days in the remdesivir group and 18 days in the placebo group. The odds ratio for improvement was higher in the remdesivir group compared with the placebo group (OR for improvement 1.50 (95\% CI 1.18-1.91), $\mathrm{p}=0.001 ; 844$ patients) at the 15th day. Mortality was not significantly different (hazard ratio 0.70 (95\% CI 0.47-1.04). The data for patients not receiving oxygen or receiving high-flow oxygen, as well as those receiving ECMO or mechanical ventilation, should be interpreted with caution due to the low number of patients recruited and followed up.

\section{Lopinavir-ritonavir}

Lopinavir-ritonavir, usually used to treat HIV infection, has been repurposed as a treatment for SARS-CoV-2 infection. In a randomised trial, no significant difference was identified in the 28-day mortality between 1596 subjects with lopinavir-ritonavir and 3376 patients in usual care (22.1\% lopinavirritonavir versus $21.3 \%$ usual care). No benefit in the risk of progression to mechanical ventilation or length of hospital stay was observed [53]. The small number of patients on invasive mechanical ventilation included does not allow clear conclusions to be made about the effect of lopinavir-ritonavir in mechanically ventilated patients.

\section{Hydroxychloroquine}

Hydroxychloroquine has been suggested as a treatment option based on its in vitro activity against SARS-CoV-2. No significant difference in 28-day mortality was identified between 1542 patients randomised to hydroxychloroquine and 3132 patients randomised to usual care $(25.7 \%$ hydroxychloroquine versus $23.5 \%$ usual care) [54]. No supportive evidence of beneficial effects on hospital stay or other outcomes was observed. These conclusions have been confirmed by a large systematic review and meta-analysis [55]. 
Take-home messages

- Remdesivir may improve the clinical outcome in severe COVID-19 patients.

- The effect of remdesivir as a stand-alone treatment has not been established.

- Combination lopinavir-ritonavir did not demonstrate clinical benefit.

- Hydroxychloroquine did not demonstrate clinical benefit.

Drugs: anti-inflammatories (R. Haynes, Oxford, UK)

R. Haynes presented data from the RECOVERY trial, a randomised controlled trial of medications (dexamethasone, lopinavir-ritonavir, tocilizumab, azithromycin, hydroxychloroquine, convalescent plasma), enrolling more than $15 \%$ of all UK hospitalised patients with COVID-19. Data from the COVACTA trial were also discussed.

\section{Dexamethasone}

The dexamethasone study arm was inspired by the long-standing contradictory outcomes of corticosteroid use in severe viral respiratory infections [56]. 2104 patients were randomised to dexamethasone 6 mg once per day for 10 days and 4321 patients to usual care. In the dexamethasone group, the incidence of death was lower than in the usual care group among patients receiving invasive mechanical ventilation $(29.3 \%$ versus $41.4 \%$; rate ratio 0.64 (95\% CI $0.51-0.81)$ ) and among those receiving oxygen without invasive mechanical ventilation (23.3\% versus 26.2\%; rate ratio 0.82 (95\% CI $0.72-0.94)$ ), but not among those who were receiving no respiratory support at randomisation (17.8\% versus $14.0 \%$; rate ratio 1.19 (95\% CI 0.92-1.55)). Benefit was also clear in patients who were treated more than 7 days after symptom onset, when inflammatory lung damage is likely to be more common. Patients in the dexamethasone group had a shorter duration of hospital stay than the usual care group (12 days versus 13 days) and a greater probability of being discharged alive within 28 days (rate ratio 1.10 (95\% CI 1.03-1.17)).

\section{Tocilizumab}

The COVACTA trial evaluated the efficacy, safety, pharmacodynamics, and pharmacokinetics of tocilizumab, an interleukin 6 (IL-6) receptor antagonist, in 450 hospitalised patients with severe COVID-19 pneumonia [57]. Despite the fact that tocilizumab-treated patients spent a week less in hospital compared with those in the placebo arm, treatment did not reduce mortality. The drawbacks of the study included broad eligibility criteria, heterogeneity of the study population, the timing of drug delivery and the timing of outcome analysis, which all limit interpretation of the results. Tocilizumab was not effective in preventing intubation or death in 243 moderately ill hospitalised patients with COVID-19. Some benefit or harm cannot be ruled out as the confidence intervals for efficacy comparisons were wide [58]. However, data published after the Congress showed a survival benefit in critically ill patients treated with tocilizumab [59].

\section{Take-home messages}

- Dexamethasone $6 \mathrm{mg}$ once daily for up to 10 days reduces 28-day mortality in patients with COVID-19 who are receiving respiratory support, i.e., invasive mechanical ventilation or oxygen (if oxygen saturations on room air are 92-94\%).

- Results of ongoing clinical trials are awaited to determine the clinical efficacy of tocilizumab in hospitalised severe COVID-19 patients.

\section{Other drugs and convalescent plasma (M. Patout, Paris, France)}

M. Patout discussed three topics regarding COVID-19 pathology and therapeutic approaches: 1) convalescent plasma; 2) thrombotic events and anticoagulation; and 3) bacterial co-infection and antibiotics.

\section{Convalescent plasma}

Convalescent plasma to treat COVID-19 disease was granted authorisation for emergency use by the US Food and Drug Administration in August 2020. M. Patout presented data from the largest clinical study of convalescent plasma therapy: plasma transfusion in severe COVID-19 disease led to clinical improvement in nine patients (36\%) by day 7 and 12 patients (48\%) by day 14 and was not associated with any clinical complications [60]. These findings are consistent with several case studies investigating the use of convalescent plasma in severe cases [61]. Despite promising results among patients with severe and/or life-threatening COVID-19 disease, several clinical questions regarding the efficacy and applicability of plasma transfusion remain, including the timing of transfusion, the volume of plasma and adjustment for body mass index, the lowest efficient titre of donor plasma, the optimal number of transfusions, the effect in early disease, and the impact on mortality. 
Thrombotic events and anticoagulation

There is overwhelming evidence that SARS-CoV-2 infection is associated with coagulopathy and increased thrombotic risk. The first European autopsy study established deep venous thrombosis in 58\% of patients and pulmonary embolism in 30\% [62]. KLOK et al. [63] reported thrombotic complications in 31\% of 84 ICU subjects: venous thromboembolism was confirmed by ultrasonography in $27 \%$, arterial thrombosis in $3.7 \%$ and pulmonary embolism in $81 \%$. The mechanisms underlying the increased prothrombotic risk in SARS-CoV-2 infection are diverse, including direct activation of coagulation by SARS-CoV-2, endothelial dysfunction, increased levels of von Willebrand factor, systemic inflammation, Toll-like receptor activation, and tissue factor pathway activation $[64,65]$.

Anticoagulation appeared to be effective in a large cohort of 4389 hospitalised COVID-19 patients [66]. Patients were categorised into three groups: 1) 1530 (34.9\%) without anticoagulation; 2) 1959 (44.6\%) with prophylactic anticoagulation; and 3) 900 (20.5\%) with therapeutic anticoagulation [62]. Those with prophylactic or therapeutic anticoagulation had a lower risk of in-hospital mortality with adjusted hazard ratios (aHR) of 0.50 (95\% CI $0.45-0.57$ ) and 0.53 (95\% CI 0.45-0.62), respectively, in comparison to no anticoagulation. Prophylactic and therapeutic dosages were associated with a lower risk of intubation compared with no anticoagulation (aHR 0.72 (95\% CI 0.58-0.89) and aHR 0.69 (95\% CI 0.51-0.94), respectively). Major bleeding was highest among patients with therapeutic anticoagulation (3.0\%) and similar for patients on prophylactic $(1.7 \%)$ or no anticoagulation $(1.9 \%)$. The authors concluded that anticoagulation in patients hospitalised with SARS-CoV-2 is associated with lower mortality and risk of intubation without a statistically significant difference between prophylactic and therapeutic dosage regimens. However, these data have not been confirmed by randomised controlled trials.

\section{Bacterial co-infections and antibiotics}

Patients with SARS-CoV-2 infection are commonly started empirically on antibacterial therapy when first hospitalised. The Michigan study reported that empiric antibacterial therapy was prescribed in 56.6\% of 1705 hospitalised SARS-CoV-2 patients within 2 days of hospital admission. However, microbiologically confirmed community-onset bacterial co-infection was established in only 3.5\% (59 out of 1705) of these patients [67]. These results are similar to other studies in UK [68] and New York [69]. Moreover, the cytokine storm, rather than bacterial sepsis, may mislead the clinician in a subset of patients, and result in clinical deterioration. The risks of unnecessary antibacterial therapy and the low rate of confirmed early bacterial co-infection in patients with SARS-CoV-2 infection suggest against routine antibacterial therapy.

Take-home messages

- The administration of convalescent plasma is a safe treatment option in severe COVID-19; its contribution as a stand-alone treatment remains elusive.

- There is a low frequency of early (up to $48 \mathrm{~h}$ ) bacterial co-infection in hospitalised COVID-19 patients and no evidence of concomitant fungal infection.

- Hospitalised COVID-19 patients are at increased thrombotic risk. Anticoagulation is reasonable and is associated with lower rates of death and intubation. There is still doubt as to whether prophylactic or therapeutic doses of anticoagulation should be administered to prevent thrombosis in hospitalised COVID-19 patients.

Hot topics in intensive care: COVID-19 - lessons learned

This session examined the most up-to-date advice at the time of the ERS Congress for the clinical management of patients with COVID-19, including respiratory support and pharmacological management. Some knowledge may have now evolved.

Clinical characteristics and outcome (A. Demoule, Paris, France)

A. Demoule began by describing demographic data. The population admitted to ICU with COVID-19 was younger compared with those usually admitted to ICU: this was because of the comparatively high mortality of older people, and because such patients were less frequently admitted to ICU due to greater disease severity [70].

Ethnicity data from the USA [71] showed that minority ethnic backgrounds were over-represented in ICU admissions (30\% African-American and 30\% Hispanic), probably due to socioeconomic and professional factors.

Regarding comorbidities: $75-85 \%$ of the ICU population was obese or overweight, 50-60\% had hypertension and $17-25 \%$ had type 2 diabetes. COPD was present in only $4-8 \%$ of patients. 
Age, male gender, quantity of organ failure, severity of hypoxaemia, morbid obesity and the number of beds in ICU departments has had an important impact on the prognosis of SARS-CoV-2 pneumonia [70].

\section{Take-home messages}

- $\quad$ Risk factors for death from severe COVID-19 include metabolic syndrome, age, and gender.

- Lung compliance is not dramatically reduced in all patients.

Respiratory support and viral transmission (S. Nava, Bologna, Italy)

S. Nava started by explaining that most patients with acute respiratory failure due to SARS-CoV-2 were managed outside the ICU [72].

In hypoxaemia unresponsive to conventional oxygen therapy, CPAP may improve intubation-free survival compared with oxygen therapy alone [73]. Response is variable, as demonstrated by an observational study of patients managed with helmet CPAP in which $21.7 \%(n=34)$ required invasive mechanical ventilation and $22.9 \%(n=36)$ died [74]. After adjustment for potential confounders, the failure rate of CPAP is not significantly different to that of other noninvasive respiratory support modalities, i.e., in the rate of mortality, ETI and length of hospital stay. Mortality rate increased with age and comorbidity class progression [41]. Improvements in oxygenation observed on proning non-intubated patients were maintained after resupination in half of patients. Further studies are warranted to ascertain the potential benefit of this technique in improving respiratory and global outcomes [75].

The second part of the presentation focused on viral transmission: $11.4 \%(n=42)$ of healthcare workers in contact with aerosol generating procedures had positive SARS-CoV-2 PCR, three of whom required hospital admission [41]. However, the infection of healthcare workers remains inconclusive; one study showed the prevalence of SARS-CoV-2 antibodies was $13.4 \%$ in a cohort of 40329 healthcare personnel, including those without direct patient contact such as administrative staff [76]. Among doctors, general practitioners were predominantly affected, probably due to close proximity to infected patients and less frequent use of personal and protective equipment (PPE), particularly in the early stages of the pandemic [41]. Although devastating for colleagues and healthcare organisations, the pandemic has reconnected healthcare workers to the communities they serve, with recognition and praise given in response to their dedication and sacrifice.

\section{Take-home messages}

- Response to CPAP in patients with SARS-CoV-2 is variable and requires further investigation to improve patient selection.

- Further investigation is required to determine SARS-CoV-2 transmission from aerosol generating procedures to inform safeguarding of healthcare workers.

Management of severe SARS-CoV-2 in resource-poor settings (A. Dondorp, Bangkok, Thailand) Nine Asian countries have been involved in a large ICU network with South American and African countries to capture important epidemiological data on ICU mortality and comorbidity. 84\% of the world's population lives in low or middle-income countries and there is no accurate data on the impact of SARS-CoV-2 on these areas.

The quality of care often depends on the healthcare setting, and many poorer countries depend on a variety of donated ventilators, which complicates management. A quarter of hospitals lack sufficient oxygen supply. Therefore, awake proning in patients with NIV has been particularly useful in these settings.

The suggested minimum level of facilities and staffing for mechanical ventilation in low-resource settings is continuous pulse oximetry while on the ventilator, 24-h presence of a member of staff with airway training, and a nurse-to-patient ratio of at least 3:1 [77].

LUS is a useful tool for lung imaging in resource-poor settings [78], because it is affordable, can be easily taught, and can help in diagnosis when testing is not available, and also in assessing "non-focal" versus "focal” lesions, or "non-compliant" or "non-recruitable” lesions.

One of the main challenges in the management of SARS-CoV-2 infection in these settings was the fact that PPE was in short supply. In addition, there was a lack of negative pressure rooms, isolation rooms, and clean water. 
The emphasis of the management of severe SARS-CoV-2 infection in resource-poor settings should focus on the availability and quality of noninvasive respiratory support, the safety of staff and the use of LUS for lung imaging.

\section{Take-home messages}

- In resource-poor settings, severe SARS-CoV-2 infection should be preferentially managed using noninvasive respiratory support.

- In resource-poor settings, safety of staff is challenging because of the lack of PPE and equipment.

How to organise research during a pandemic (M. Gong, Bronx, USA)

M. Gong presented an algorithm for the organisation of clinical research used in Montefiore, Italy, during the pandemic. Each trial was assigned to a senior clinical investigator, a non-clinical research fellow who coordinated all studies, and two research groups: 1) experienced critical care research coordinators that screened and enrolled patients, collected samples and performed hospital-based study procedures, and 2) volunteer research coordinators (medical students) who performed data entry and query resolution. All other clinical research was suspended.

Clinical research during a pandemic is possible, but challenging. It is important to adapt infrastructures to pandemic standards, including expediting regulatory approvals. Because of the urgency of trials, it also requires national leadership. Pandemic research became as urgent as pandemic clinical care: human subject review and approval was centralised and expedited; regulatory paperwork was reviewed and signed in a rapid fashion; research pharmacy and clinical pharmacy collaborated and were able to implement trials within days; and alternatives to written consent were accepted, e.g. electronic signatures, or in the USA, verbal consent and electronic signature.

\section{Take-home messages}

- Clinical research during a pandemic is possible by adapting infrastructure to pandemic standards and expediting regulatory approvals.

- It is important to take into account the tension some physicians feel between clinical care and clinical trials during a pandemic.

Acknowledgements: The authors would like to thank the organising committee of the ERS International Congress 2020 and the speakers in the reported sessions: C. Agerstrand, J. Beigel, L.D.J. Bos, A. Demoule, A. Dondorp, M. Gong, R. Heynes, M. Kokosi, S. Nava, P. Navalesi, V. Poletti, M. Prokop, C. Ravaglia and N. Sverzellati.

Provenance: Commissioned article, peer reviewed.

Conflict of interest: C. Rolland-Debord has nothing to disclose. A. D'Haenens has nothing to disclose. L. Mendiluce has nothing to disclose. L. Spurr has nothing to disclose. S. Konda has nothing to disclose. R. Cherneva has nothing to disclose. E. Lhuillier has nothing to disclose. L. Heunks has nothing to disclose. M. Patout reports personal fees from Resmed and Philips Respironics, grants and nonfinancial support from Fisher \& Paykel, nonfinancial support and personal fees from Asten, research grants from B\&D Electromedical and Fisher \& Paykel, and personal fees and nonfinancial support from Chiesi, outside the submitted work.

\section{References}

1 Kearney SE, Davies CW, Davies RJ, et al. Computed tomography and ultrasound in parapneumonic effusions and empyema. Clin Radiol 2000; 55: 542-547.

2 Yang PC, Luh KT, Chang DB, et al. Value of sonography in determining the nature of pleural effusion: analysis of 320 cases. AJR Am J Roentgenol 1992; 159: 29-33.

3 Aublanc M, Perinel S, Guerin C. Acute respiratory distress syndrome mimics: the role of lung biopsy. Curr Opin Crit Care 2017; 23: 24-29.

4 Schnabel RM, van der Velden K, Osinski A, et al. Clinical course and complications following diagnostic bronchoalveolar lavage in critically ill mechanically ventilated patients. BMC Pulm Med 2015; 15: 107.

5 Poletti V, Poletti G, Murer B, et al. Bronchoalveolar lavage in malignancy. Semin Respir Crit Care Med 2007; 28: 534-545.

6 Messika J, Darmon M, Mal $\mathrm{H}$, et al. Etiologies and outcomes of acute respiratory failure in solid organ transplant recipients: insight into the EFRAIM multicenter cohort. Transplant Proc 2020; 52: 2980-2987. 
7 Guerin C, Bayle F, Leray V, et al. Open lung biopsy in nonresolving ARDS frequently identifies diffuse alveolar damage regardless of the severity stage and may have implications for patient management. Intensive Care Med 2015; 41: 222-230.

8 Almotairi A, Biswas S, Shahin J. The role of open lung biopsy in critically ill patients with hypoxic respiratory failure: a retrospective cohort study. Can Respir J 2016; 2016: 8715024.

9 Libby LJ, Gelbman BD, Altorki NK, et al. Surgical lung biopsy in adult respiratory distress syndrome: a meta-analysis. Ann Thorac Surg 2014; 98: 1254-1260.

10 O'Brien JD, Ettinger NA, Shevlin D, et al. Safety and yield of transbronchial biopsy in mechanically ventilated patients. Crit Care Med 1997; 25: 440-446.

11 Cooley J, Swank ZG, Nakahodo AAA, et al. Safety and utility of transbronchial lung cryobiopsy in mechanically ventilated patients with ARDS. Am J Respir Crit Care Med 2019; 199: Suppl., A1401.

12 Brusasco C, Santori G, Bruzzo E, et al. Quantitative lung ultrasonography: a putative new algorithm for automatic detection and quantification of B-lines. Crit Care 2019; 23: 288.

13 Bouhemad B, Brisson $\mathrm{H}$, Le-Guen $\mathrm{M}$, et al. Bedside ultrasound assessment of positive end-expiratory pressure-induced lung recruitment. Am J Respir Crit Care Med 2011; 183: 341-347.

14 Yusuf GT, Wong A, Rao D, et al. The use of contrast-enhanced ultrasound in COVID-19 lung imaging. J Ultrasound 2020; in press [https://doi.org/10.1007/s40477-020-00517-z].

15 Mooij-Kalverda KA, Wijmans L, Bos L, et al. Confocal laser endomicroscopy (CLE) in patients with acute respiratory failure on the ICU. Eur Respir J 2019; 54: Suppl. 63, PA3170.

16 Baddley JW, Winthrop KL, Chen L, et al. Non-viral opportunistic infections in new users of tumour necrosis factor inhibitor therapy: results of the SAfety Assessment of Biologic ThERapy (SABER) study. Ann Rheum Dis 2014; 73: 1942-1948.

17 van Oort PM, Povoa P, Schnabel R, et al. The potential role of exhaled breath analysis in the diagnostic process of pneumonia-a systematic review. J Breath Res 2018; 12: 024001.

18 Pendleton KM, Erb-Downward JR, Bao Y, et al. Rapid pathogen identification in bacterial pneumonia using real-time metagenomics. Am J Respir Crit Care Med 2017; 196: 1610-1612.

19 Langelier C, Kalantar KL, Moazed F, et al. Integrating host response and unbiased microbe detection for lower respiratory tract infection diagnosis in critically ill adults. Proc Natl Acad Sci USA 2018; 115: E12353-E12362.

20 Dickson RP, Schultz MJ, van der Poll T, et al. Lung microbiota predict clinical outcomes in critically ill patients. Am J Respir Crit Care Med 2020; 201: 555-563.

21 Morrell ED, Radella F, II, Manicone AM, et al. Peripheral and alveolar cell transcriptional programs are distinct in acute respiratory distress syndrome. Am J Respir Crit Care Med 2018; 197: 528-532.

22 Villar J, Slutsky AS. GOLDEN anniversary of the acute respiratory distress syndrome: still much work to do! Curr Opin Crit Care 2017; 23: 4-9.

23 Force ADT, Ranieri VM, Rubenfeld GD, et al. Acute respiratory distress syndrome: the Berlin definition. JAMA 2012; 307: 2526-2533.

24 Petrosyan F, Culver DA, Reddy AJ. Role of bronchoalveolar lavage in the diagnosis of acute exacerbations of idiopathic pulmonary fibrosis: a retrospective study. BMC Pulm Med 2015; 15: 70.

25 Ravaglia C, Gurioli C, Casoni G, et al. Diagnostic role of rapid on-site cytologic examination (ROSE) of broncho-alveolar lavage in ALI/ARDS. Pathologica 2012; 104: 65-69.

26 Arcadu A, Moua T. Bronchoscopy assessment of acute respiratory failure in interstitial lung disease. Respirology 2017; 22: 352-359.

27 de Prost N, Pham T, Carteaux G, et al. Etiologies, diagnostic work-up and outcomes of acute respiratory distress syndrome with no common risk factor: a prospective multicenter study. Ann Intensive Care 2017; 7 : 69.

28 Philipponnet C, Cassagnes L, Pereira B, et al. Diagnostic yield and therapeutic impact of open lung biopsy in the critically ill patient. PLOS One 2018; 13: e0196795.

29 Araya J, Kawabata Y, Jinho P, et al. Clinically occult subpleural fibrosis and acute interstitial pneumonia a precursor to idiopathic pulmonary fibrosis? Respirology 2008; 13: 408-412.

30 Zafrani L, Lemiale V, Lapidus N, et al. Acute respiratory failure in critically ill patients with interstitial lung disease. PLoS One 2014; 9: e104897.

31 Akira M, Kozuka T, Yamamoto S, et al. Computed tomography findings in acute exacerbation of idiopathic pulmonary fibrosis. Am J Respir Crit Care Med 2008; 178: 372-378.

32 Sokai A, Tanizawa K, Handa T, et al. Asymmetry in acute exacerbation of idiopathic pulmonary fibrosis. ERJ Open Res 2017; 3: 00036-2016.

33 Peek GJ, Mugford M, Tiruvoipati R, et al. Efficacy and economic assessment of conventional ventilatory support versus extracorporeal membrane oxygenation for severe adult respiratory failure (CESAR): a multicentre randomised controlled trial. Lancet 2009; 374: 1351-1363.

34 Combes A, Hajage D, Capellier G, et al. Extracorporeal membrane oxygenation for severe acute respiratory distress syndrome. N Engl J Med 2018; 378: 1965-1975. 

extracorporeal membrane oxygenation for acute respiratory failure. Am J Respir Crit Care Med 2016; 193: 527-533.

36 Biscotti M, Lee A, Basner RC, et al. Hybrid configurations via percutaneous access for extracorporeal membrane oxygenation: a single-center experience. ASAIO J 2014; 60: 635-642.

37 Chicotka S, Rosenzweig EB, Brodie D, et al. The "Central Sport Model": extracorporeal membrane oxygenation using the innominate artery for smaller patients as bridge to lung transplantation. ASAIO J 2017; 63: e39-e44.

38 Toyoda Y, Hanibuchi M, Kishi J, et al. Clinical features and outcome of acute exacerbation of interstitial pneumonia associated with connective tissue disease. J Med Invest 2016; 63: 294-299.

39 Mollica C, Paone G, Conti V, et al. Mechanical ventilation in patients with end-stage idiopathic pulmonary fibrosis. Respiration 2010; 79: 209-215.

40 Gungor G, Tatar D, Salturk C, et al. Why do patients with interstitial lung diseases fail in the ICU? A 2-center cohort study. Respir Care 2013; 58: 525-531.

41 Franco C, Facciolongo N, Tonelli R, et al. Feasibility and clinical impact of out-of-ICU noninvasive respiratory support in patients with COVID-19-related pneumonia. Eur Respir J 2020; 56: 2002130.

42 Tobin MJ. Basing respiratory management of COVID-19 on physiological principles. Am J Respir Crit Care Med 2020; 201: 1319-1320.

43 Pasin L, Sella N, Correale C, et al. Regional COVID-19 network for coordination of SARS-CoV-2 outbreak in Veneto, Italy. J Cardiothorac Vasc Anesth 2020; 34: 2341-2345.

44 Schultz MJ. High versus low PEEP in non-recruitable collapsed lung tissue: possible implications for patients with COVID-19. Lancet Respir Med 2020; 8: e44.

45 Sella N, Zarantonello F, Andreatta G, et al. Positive end-expiratory pressure titration in COVID-19 acute respiratory failure: electrical impedance tomography vs. PEEP/FiO2 tables. Crit Care 2020; $24: 540$.

46 van der Zee P, Somhorst P, Endeman $\mathrm{H}$, et al. Electrical impedance tomography for positive end-expiratory pressure titration in COVID-19-related acute respiratory distress syndrome. Am J Respir Crit Care Med 2020; 202: $280-284$.

47 Spiezia L, Boscolo A, Poletto F, et al. COVID-19-related severe hypercoagulability in patients admitted to intensive care unit for acute respiratory failure. Thromb Haemost 2020; 120: 998-1000.

48 Jose RJ, Manuel A. COVID-19 cytokine storm: the interplay between inflammation and coagulation. Lancet Respir Med 2020; 8: e46-e47.

49 Zarantonello F, Andreatta G, Sella N, et al. Prone position and lung ventilation and perfusion matching in acute respiratory failure due to COVID-19. Am J Respir Crit Care Med 2020; 202: 278-279.

50 Schmidt M, Hajage D, Lebreton G, et al. Extracorporeal membrane oxygenation for severe acute respiratory distress syndrome associated with COVID-19: a retrospective cohort study. Lancet Respir Med 2020; 8 1121-1131.

51 Shekar K, Slutsky AS, Brodie D. ECMO for severe ARDS associated with COVID-19: now we know we can, but should we? Lancet Respir Med 2020; 8: 1066-1068.

52 Beigel JH, Tomashek KM, Dodd LE, et al. Remdesivir for the treatment of Covid-19 - final report. N Engl J Med 2020; 383: 1813-1826.

53 RECOVERY Collaborative Group. Lopinavir-ritonavir in patients admitted to hospital with COVID-19 (RECOVERY): a randomised, controlled, open-label, platform trial. Lancet 2020; 396: 1345-1352.

54 RECOVERY Collaborative Group, Horby P, Mafham M, et al. Effect of hydroxychloroquine in hospitalized patients with Covid-19. N Engl J Med 2020; 383: 2030-2040.

55 Fiolet T, Guihur A, Rebeaud ME, et al. Effect of hydroxychloroquine with or without azithromycin on the mortality of coronavirus disease 2019 (COVID-19) patients: a systematic review and meta-analysis. Clin Microbiol Infect 2021; 27: 19-27.

56 RECOVERY Collaborative Group, Horby P, Lim WS, et al. Dexamethasone in hospitalized patients with Covid-19. N Engl J Med 2021; 384: 693-704.

57 Stone JH, Frigault MJ, Serling-Boyd NJ, et al. Efficacy of tocilizumab in patients hospitalized with Covid-19. N Engl J Med 2020; 383: 2333-2344.

58 Furlow B. COVACTA trial raises questions about tocilizumab's benefit in COVID-19. Lancet Rheumatol 2020; 2: e592.

59 REMAP-CAP Investigators, Gordon AC, Mouncey PR, et al. Interleukin-6 receptor antagonists in critically ill patients with Covid-19. N Engl J Med 2021; 384: 1491-1502.

60 Salazar E, Perez KK, Ashraf M, et al. Treatment of coronavirus disease 2019 (COVID-19) patients with convalescent plasma. Am J Pathol 2020; 190: 1680-1690.

61 Duan K, Liu B, Li C, et al. Effectiveness of convalescent plasma therapy in severe COVID-19 patients. Proc Natl Acad Sci USA 2020; 117: 9490-9496.

62 Wichmann D, Sperhake JP, Lutgehetmann M, et al. Autopsy findings and venous thromboembolism in patients with COVID-19: a prospective cohort study. Ann Intern Med 2020; 173: 268-277. 
63 Klok FA, Kruip M, van der Meer NJM, et al. Incidence of thrombotic complications in critically ill ICU patients with COVID-19. Thromb Res 2020; 191: 145-147.

64 Giannis D, Ziogas IA, Gianni P. Coagulation disorders in coronavirus infected patients: COVID-19, SARS-CoV-1, MERS-CoV and lessons from the past. J Clin Virol 2020; 127: 104362.

65 Li H, Liu L, Zhang D, et al. SARS-CoV-2 and viral sepsis: observations and hypotheses. Lancet 2020; 395: 1517-1520.

66 Nadkarni GN, Lala A, Bagiella E, et al. Anticoagulation, bleeding, mortality, and pathology in hospitalized patients with COVID-19. J Am Coll Cardiol 2020; 76: 1815-1826.

67 Vaughn VM, Gandhi TN, Petty LA, et al. Empiric antibacterial therapy and community-onset bacterial coinfection in patients hospitalized with coronavirus disease 2019 (COVID-19): a multi-hospital cohort study. Clin Infect Dis 2021; 72: e533-e541.

68 Hughes $\mathrm{S}$, Troise $\mathrm{O}$, Donaldson $\mathrm{H}$, et al. Bacterial and fungal coinfection among hospitalized patients with COVID-19: a retrospective cohort study in a UK secondary-care setting. Clin Microbiol Infect 2020; 26: 1395-1399.

69 Nori P, Cowman K, Chen V, et al. Bacterial and fungal coinfections in COVID-19 patients hospitalized during the New York City pandemic surge. Infect Control Hosp Epidemiol 2021; 42: 84-88.

70 Grasselli G, Zangrillo A, Zanella A, et al. Baseline characteristics and outcomes of 1591 patients infected with SARS-CoV-2 admitted to ICUs of the Lombardy Region, Italy. JAMA 2020; 323: 1574-1581.

71 Gupta S, Hayek SS, Wang W, et al. Factors associated with death in critically ill patients with coronavirus disease 2019 in the US. JAMA Intern Med 2020; 180: 1436-1447.

72 Suleyman G, Fadel RA, Malette KM, et al. Clinical characteristics and morbidity associated with coronavirus disease 2019 in a series of patients in Metropolitan Detroit. JAMA Netw Open 2020; 3: e2012270.

73 Oranger M, Gonzalez-Bermejo J, Dacosta-Noble P, et al. Continuous positive airway pressure to avoid intubation in SARS-CoV-2 pneumonia: a two-period retrospective case-control study. Eur Respir J 2020; 56: 2001692.

74 Aliberti S, Radovanovic D, Billi F, et al. Helmet CPAP treatment in patients with COVID-19 pneumonia: a multicentre cohort study. Eur Respir J 2020; 56: 2001935.

75 Coppo A, Bellani G, Winterton D, et al. Feasibility and physiological effects of prone positioning in non-intubated patients with acute respiratory failure due to COVID-19 (PRON-COVID): a prospective cohort study. Lancet Respir Med 2020; 8: 765-774.

76 Moscola J, Sembajwe G, Jarrett M, et al. Prevalence of SARS-CoV-2 antibodies in health care personnel in the New York City Area. JAMA 2020; 324: 893-895.

77 Inglis R, Ayebale E, Schultz MJ. Optimizing respiratory management in resource-limited settings. Curr Opin Crit Care 2019; 25: 45-53.

78 Schultz MJ, Sivakorn C, Dondorp AM. Challenges and opportunities for lung ultrasound in novel coronavirus disease (COVID-19). Am J Trop Med Hyg 2020; 102: 1162-1163.

79 Patout M, Fresnel E, Lujan M, et al. Recommended approaches to minimize aerosol dispersion of SARS-CoV-2 during noninvasive ventilatory support can cause ventilator performance deterioration: a benchmark comparative study. Chest 2021; 160: 175-186. 